\title{
DECOMPOSITION OF THE TENSOR PRODUCT OF COMPLETE GRAPHS INTO CYCLES OF LENGTHS 3 AND 6
}

\author{
P. Paulraja And R. SRImathi \\ Department of Mathematics \\ Kalasalingam Academy of Research and Education \\ Krishnankoil-626126, India \\ e-mail: ppraja56@gmail.com \\ gsrimathi66@gmail.com
}

\begin{abstract}
By a $\left\{C_{3}^{\alpha}, C_{6}^{\beta}\right\}$-decomposition of a graph $G$, we mean a partition of the edge set of $G$ into $\alpha$ cycles of length 3 and $\beta$ cycles of length 6 . In this paper, necessary and sufficient conditions for the existence of a $\left\{C_{3}^{\alpha}, C_{6}^{\beta}\right\}$ decomposition of $\left(K_{m} \times K_{n}\right)(\lambda)$, where $\times$ denotes the tensor product of graphs and $\lambda$ is the multiplicity of the edges, is obtained. In fact, we prove that for $\lambda \geq 1, m, n \geq 3$ and $(m, n) \neq(3,3)$, a $\left\{C_{3}^{\alpha}, C_{6}^{\beta}\right\}$-decomposition of $\left(K_{m} \times K_{n}\right)(\lambda)$ exists if and only if $\lambda(m-1)(n-1) \equiv 0(\bmod 2)$ and $3 \alpha+6 \beta=\frac{\lambda m(m-1) n(n-1)}{2}$.
\end{abstract}

Keywords: cycle decomposition, tensor product.

2010 Mathematics Subject Classification: 05B30, 05C70.

\section{REFERENCES}

[1] B. Alspach and H. Gavlas, Cycle decompositions of $K_{n}$ and $K_{n}-I$, J. Combin. Theory Ser. B 81 (2001) 77-99.

doi:10.1006/jctb.2000.1996

[2] J. Asplund, J. Chaffee and J.M. Hammer, Decomposition of a complete bipartite multigraph into arbitrary cycle sizes, Graphs Combin. 33 (2017) 715-728. doi:10.1007/s00373-017-1817-0

[3] A.M. Assaf, Modified group divisible designs, Ars Combin. 29 (1990) 13-20.

[4] A.M. Assaf, An application of modified group divisible designs, J. Combin. Theory Ser. A 68 (1994) 152-168. doi:10.1016/0097-3165(94)90095-7 
[5] A.M. Assaf, Modified group divisible designs with block size 4 and $\lambda>1$, Australas. J. Combin. 16 (1997) 229-238.

[6] A.M. Assaf and R. Wei, Modified group divisible designs with block size 4 and $\lambda=1$, Discrete Math. 195 (1999) 15-25. doi:10.1016/S0012-365X(98)00161-7

[7] M.A. Bahmanian and M. Šajna, Decomposing complete equipartite multigraphs into cycles of variable lengths: The Amalgamation-detachment approach, J. Combin. Des. 24 (2016) 165-183. doi: $10.1002 /$ jcd. 21419

[8] R. Balakrishnan, J.-C. Bermond, P. Paulraja and M.-L. Yu, On Hamilton cycle decompositions of the tensor product of complete graphs, Discrete Math. 268 (2003) 49-58. doi:10.1016/S0012-365X(02)00680-5

[9] R. Balakrishnan and K. Ranganathan, A Textbook of Graph Theory, $2^{\text {nd }}$ Ed. (Springer, New York, 2012). doi:10.1007/978-1-4614-4529-6

[10] E.J. Billington, Decomposing complete tripartite graphs into cycles of lengths 3 and 4, Discrete Math. 197/198 (1999) 123-135. doi:10.1016/S0012-365X(99)90049-3

[11] E.J. Billington and N.J. Cavenagh, Sparse graphs which decompose into closed trails of arbitrary lengths, Graphs Combin. 24 (2008) 129-147. doi:10.1007/s00373-008-0783-y

[12] E.J. Billington, D.G. Hoffman and B.M. Maenhaut, Group divisible pentagon systems, Util. Math. 55 (1999) 211-219.

[13] D. Bryant, D. Horsley and W. Pettersson, Cycle decompositions V: Complete graphs into cycles of arbitrary lengths, Proc. Lond. Math. Soc. (3) 108 (2014) 1153-1192. doi:10.1112/plms/pdt051

[14] D. Bryant, D. Horsley, B. Maenhaut and B.R. Smith, Decompositions of complete multigraphs into cycles of varying lengths, J. Combin. Theory Ser. B 129 (2018) 79-106. doi:10.1016/j.jctb.2017.09.005

[15] M. Buratti, H. Cao, D. Dai and T. Traetta, A complete solution to the existence of $(k, \lambda)$-cycle frames of type $g^{u}, \mathrm{~J}$. Combin. Des. 25 (2017) 197-230. doi: $10.1002 /$ jcd. 21523

[16] C.C. Chou, C.M. Fu and W.C. Huang, Decomposition of $K_{m, n}$ into short cycles, Discrete Math. 197/198 (1999) 195-203. doi:10.1016/S0012-365X(99)90063-8

[17] C.C. Chou and C.M. Fu, Decomposition of $K_{m, n}$ into 4-cycles and 2t-cycles, J. Comb. Optim. 14 (2007) 205-218. doi:10.1007/s10878-007-9060-x 
[18] C.M. Fu, K.C. Huang and M. Mishima, Decomposition of complete bipartite graphs into cycles of distinct even lengths, Graphs Combin. 32 (2016) 1397-1413. doi:10.1007/s00373-015-1664-9

[19] S. Ganesamurthy and P. Paulraja, Decompositions of complete tripartite graphs into cycles of lengths 3 and 6, Australas. J. Combin. 73 Part 1, to appear.

[20] H. Hanani, Balanced incomplete block designs and related designs, Discrete Math. 11 (1975) 255-369. doi:10.1016/0012-365X(75)90040-0

[21] D.G. Hoffman, C.C. Lindner and C.A. Rodger, On the construction of odd cycle systems, J. Graph Theory 13 (1989) 417-426. doi:10.1002/jgt.3190130405

[22] M.H. Huang and H.L. Fu, (4,5)-cycle systems of complete multipartite graphs, Taiwanese J. Math. 16 (2012) 999-1006. doi:10.11650/twjm/1500406672

[23] C.C. Lindner and C.A. Rodger, Design Theory, $2^{\text {nd }}$ Ed. (CRC Press, Boca Raton, 2009).

[24] A.C.H. Ling and C.J. Colbourn, Modified group divisible designs with block size four, Discrete Math. 219 (2000) 207-221. doi:10.1016/S0012-365X(99)00342-8

[25] R.S. Manikandan and P. Paulraja, $C_{p}$-decompositions of some regular graphs, Discrete Math. 306 (2006) 429-451. doi:10.1016/j.disc.2005.08.006

[26] R.S. Manikandan and P. Paulraja, $C_{5}$-decompositions of the tensor product of complete graphs, Australas. J. Combin. 37 (2007) 285-293.

[27] R.S. Manikandan and P. Paulraja, $C_{7}$-decompositions of the tensor product of complete graphs, Discuss. Math. Graph Theory 37 (2017) 523-535. doi:10.7151/dmgt.1936

[28] R.S. Manikandan and P. Paulraja, Hamiltonian decompositions of the tensor product of a complete graph and a complete bipartite graphs, Ars Combin. 80 (2006) 33-44.

[29] R.S. Manikandan and P. Paulraja, Hamilton cycle decompositions of the tensor product of complete multipartite graphs, Discrete Math. 308 (2008) 3586-3606. doi:10.1016/j.disc.2007.07.020

[30] R.S. Manikandan and P. Paulraja, Hamilton cycle decompositions of the tensor products of complete bipartite graphs and complete multipartite graphs, Discrete Math. 310 (2010) 2776-2789. doi:10.1016/j.disc.2010.05.034

[31] R.S. Manikandan, P. Paulraja and S. Sivasankar, Directed Hamilton cycle decompositions of the tensor product of symmetric digraphs, Ars Combin. 98 (2011) 379-386.

[32] A. Muthusamy and A. Shanmuga Vadivu, Cycle frames of complete multipartite multigraphs-III, J. Combin. Des. 22 (2014) 473-487. doi:10.1002/jcd. 21373 
[33] P. Paulraja and S. Sampath Kumar, Resolvable even cycle decompositions of the tensor product of complete graphs, Discrete Math. 311 (2011) 1841-1850.

doi:10.1016/j.disc.2011.04.028

[34] P. Paulraja and S. Sampath Kumar, Closed trail decompositions of some classes of regular graphs, Discrete Math. 312 (2012) 1353-1366.

doi:10.1016/j.disc.2011.12.015

[35] P. Paulraja and S. Sivasankar, Directed Hamilton cycle decompositions of the tensor products of symmetric digraphs, Graphs Combin. 25 (2009) 571-581. doi:10.1007/s00373-009-0866-4

[36] M. Šajna, Cycle decompositions III: Complete graphs and fixed length cycles, J. Combin. Des. 10 (2002) 27-78. doi: $10.1002 /$ jcd. 1027

Received 3 March 2018

Revised 3 October 2018

Accepted 3 October 2018 